\title{
Pancreatite aguda sem etiologia aparente: a microlitíase deve ser pesquisada?
}

\section{Unexplained acute pancreatitis: the microlithiasis must be searched?}

José Celso Ardengh, SObed-SP1; Djalma Ernesto Coelho, ACBC-RJ²; José Sebastião dos Santos, tCBC-SP1; José Luiz Pimenta Módena, TCBC-SP1; José Marcus Raso Eulalio TCBC-RJ33; José Flávio Coelho, SOBED-RJ3

\section{INTRODUÇÃO}

mportante função da glândula pancreática é a secreção de enzimas lipolíticas, proteolíticas e de amilase, necessárias à digestão de nutrientes no intestino ${ }^{1}$. A autodigestão pancreática não ocorre em condições normais, pois existem mecanismos protetores: secreção de enzimas proteolíticas na forma inativa (zimogênio), sistemas de estocagem especializados nos ácinos pancreáticos e a presença de inibidores sistêmicos das proteases ${ }^{2}$. Alguns fatores podem causar a interrupção desse mecanismo de salvaguarda levando à pancreatite aguda (PA), resultante da ativação inapropriada de enzimas e substâncias bioativas ${ }^{2}$.

Define-se PA como processo inflamatório agudo com variável envolvimento de outros órgãos próximos ou à distância ${ }^{2}$. Sua incidência varia de 2 a 50/100.000 habitantes $^{3}$ e parece ter crescido a partir da década de 80, provavelmente pelo aumento da ingestão alcoólica entre jovens e da formação de cálculos biliares em algumas áreas $^{4}$. Essas duas etiologias respondem por cerca de $80 \%$ dos casos de PA².

Felizmente, muitos casos de PA são leves e autolimitados. Todavia cerca de $20 \%$ manifestam-se como pancreatite aguda grave segundo a classificação de Atlanta. A pancreatite necrosante é uma forma de pancreatite grave associada com maior incidência de complicações infecciosas locais e sistêmicas, e com maior mortalidade. é grave e, associada a complicações locais ou sistêmicas, pode ser fatal 2,5 .

\section{Etiologia da pancreatite aguda}

Muitas etiologias têm sido atribuídas à PA, porém em alguns episódios, a identificação da causa específica pode ser especialmente difícil ${ }^{6}$. Uma vez que a etiologia de um determinado episódio é comprovada e eliminada, a tendência é a evolução com menos complicações, e a prevenção de novos surtos ${ }^{1,7}$.

O consumo abusivo de bebidas alcoólicas e a presença de cálculos biliares são causas habituais da PA ${ }^{1,2}$ e responsáveis por 50 a $80 \%$ dos casos. A primeira é mais freqüente no sexo masculino e a última no feminino ${ }^{1}$. A percepção dessas condições pode ser obtida facilmente pela história do doente (uso de bebidas alcoólicas) ou US na colecistolitíase ${ }^{4}$.

A litíase biliar é responsável por aproximadamente $60 \%$ dos casos de $\mathrm{PA}^{8}$ devido, em teoria, a cálculos encravados transitoriamente na papila duodenal ${ }^{9}$. De fato, em pacientes com pancreatite aguda, cálculos são freqüentemente encontrados na vesícula biliar, no colédoco durante a exploração cirúrgica em doentes com PA e achados nas fezes tamisadas, nas primeiras 24 horas de evolução do episódio agudo, em mais de $90 \%$ dos pacientes ${ }^{9,10}$.

Analisando-se os casos de PA biliar, 80\% dos cálculos são identificados pelos US após a resolução do ataque $^{11} ; 10 \%$ visualizados pela CPER $^{12}$ e/ou CPRM ${ }^{13,14} \mathrm{e}$ os remanescentes detectados durante a laparotomia ou no exame post-mortem. ${ }^{10,15,16}$. Seis a $8 \%$ dos indivíduos com cálculos biliares apresentam episódios de $\mathrm{PA}^{17}$ e naqueles com microcálculos a taxa atinge $22 \%{ }^{18}$.

Em alguns doentes, o surto de PA relaciona-se a hipertrigliceridemia, hipercalcemia, reações aos fármacos, trauma abdominal, trauma operatório, ou à CPER antes executada ${ }^{19,20}$.

\section{Diagnóstico Clínico e Laboratorial}

O diagnóstico se baseia no quadro de dor no andar superior do abdome, náuseas, vômitos, dor à palpação abdominal, e elevação das enzimas séricas amilase e lipase acima de três vezes o limite superior da normalidade ${ }^{21,22}$.

\section{Diagnóstico por Imagem}

A radiografia simples de abdome, o US, a tomografia computadorizada (TC), a ressonância magnética (RM), a CPER e a ecoendoscopia (EE), auxiliam no diagnóstico etiológico, identificam complicações locais ou à distância e avaliam a gravidade da doença 2, 23-27.

A radiografia simples do abdome mostra evidências indiretas da inflamação mas é geralmente inespecífica para o diagnóstico etiológico. Em raros casos podem ser vistos cálculos radiopacos na topografia da vesícula biliar (Figura 1). A presença de (1) íleo localizado (alça sentinela) ou (2) generalizado, (3) o apagamento do cólon, (4) o sinal do halo renal, ou ainda (5) a presença de gás no retroperitônio, podem mostrar a gravidade da doença ${ }^{28}$.

1. Departamento de Anatomia e Cirurgia da Faculdade de Medicina de Ribeirão Preto - Universidade de São Paulo. 2. Departamento de Cirurgia da Universidade Estácio de Sá do Rio de Janeiro - UNESA. 3. Departamento de Cirurgia da Universidade Federal do Rio de Janeiro - UFRJ. 
O US pode ser usado no diagnóstico da PA, mas sua sensibilidade pode ser freqüentemente prejudicada pela grande quantidade de gases no sistema digestório. A tomografia computadorizada é mais precisa que o US para este fim $^{29}$. O US tem alta acurácia na descoberta de cálculos na vesícula biliar fora do surto da PA ${ }^{11}$. Durante o episódio agudo, seu poder diagnóstico reduz-se ${ }^{30}$, embora Parodi et al. tenham relatado $96,6 \%$ de diagnósticos positivos ${ }^{31}$. Não obstante, (1) a identificação de cálculos com dimensão inferior a 3 mm e (2) a visualização do colédoco terminal podem não ser possíveis com aparelhos convencionais de ultra-som. ${ }^{32}$. Mesmo assim o US é usado como exame para avaliação inicial por imagem por ser barato, de fácil execução (móvel), reprodutível e disponível ${ }^{29}$. O US mostra o aumento do volume pancreático (edema), presença de líquido livre na cavidade, cálculos vesiculares e dilatação da via biliar ${ }^{2}$. Sua sensibilidade e especificidade no diagnóstico da colestase extra-hepática são de 94\% e 100\%, respectivamente ${ }^{33}$. O local da obstrução biliar é diagnosticado em mais de $90 \%$ dos doentes e a causa em $71 \%$ dos $\operatorname{casos}^{33}$, ressaltar que a presença de íleo paralítico ou obesidade dificultam o exame do pâncreas em 25 a 50\% dos casos de $\mathrm{PA}^{23}$. Por outro lado outros trabalhos demonstram sensibilidade de $45 \%{ }^{30}, 47 \%{ }^{34}$ e $50 \%{ }^{25}$ para o diagnóstico de cálculos como etiologia do episódio de PA (Figura 2).

A TC é considerada exame útil na avaliação de pacientes com PA, pois possibilita a observação completa de todo o abdome e retroperitônio ${ }^{35}$ e no exame contrastado, a ausência de captação do contraste identifica áreas de necrose. A acurácia global da TC no diagnóstico da colestase extra-hepática varia de $87 \%$ a $98 \%{ }^{33}$. As desvantagens são: o custo, a necessidade de transporte do doente, a reprodutibilidade limitada, a exposição à radiação ionizante e a injeção de contraste que pode agravar a função renal ${ }^{22,23}$. No entanto o diagnóstico da causa, principalmente se ela for biliar, pela TC deixa muito a desejar com cifras que se aproximam de $47 \%{ }^{34}$.

O estudo de Balthazar et al. ${ }^{35}$ descreveu critérios tomográficos que passaram a ser empregados para estabelecer o prognóstico da PA, dentre eles citam-se: (1) aumento do tamanho do pâncreas, (2) inflamação peripancreática, (3) coleções líquidas e (4) ausência de captação do contraste na topografia do parênquima pancreático indicando presença de necrose.

A CPER, em muitas ocasiões, pode ser de extrema valia para o diagnóstico. Todavia é invasiva, e não é isenta de riscos e complicações para o doente ${ }^{32,36}$. A colangiopancreatografia por ressonância magnética (CPRM) nos oferece excelentes imagens da via bilio-pancreática sem a injeção de contraste ou exposição à radiação. Apesar de apresentar sensibilidade e especificidade superiores a 90\% no diagnóstico de cálculos biliares $^{13,37}$, apenas um estudo avaliou o seu papel na PA. Infelizmente os resultados desse estudo englobam 30 doentes com PC, sendo difícil definir sua importância na PA ${ }^{38}$.

\section{Pancreatite aguda sem causa aparente}

Não obstante o referido anteriormente, em muitos episódios de PA não se identifica a causa, mesmo após

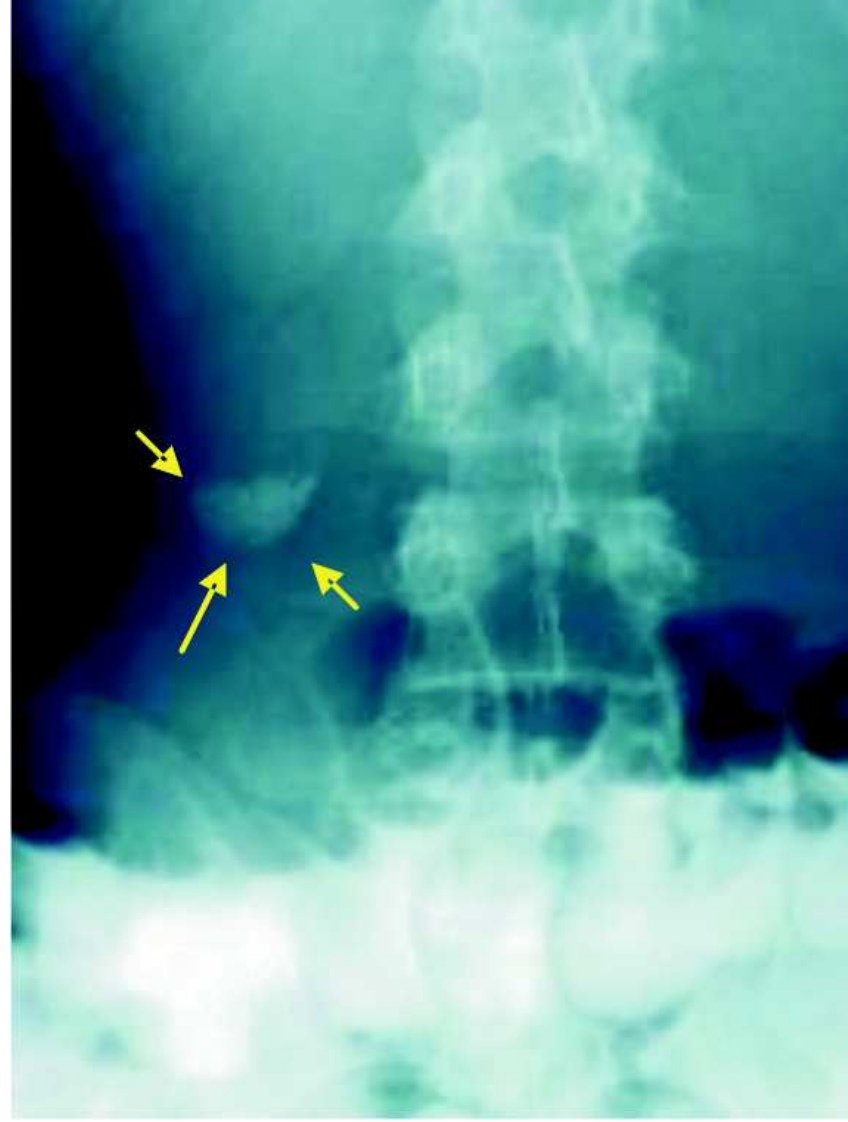

Figura 1 - $\quad$ Imagem de Rx simples de abdome onde observamos a presença de microcálculos no interior da vesícula biliar (setas amarelas).



Figura 2 - Imagem de US convencional demonstrando a presença de múltiplas áreas hiperecóicas (microcálculos) que ao se aglomerarem no interior da vesícula biliar apresentam sombra acústica.

serem colhidos e analisados em conjunto (1) a história clínica e (2) o exame físico bem feito, e (3) os exames complementares disponíveis: US, dosagem de triglicérides e cálcio que se revelam normais. Tais episódios são rotulados como "sem causa aparente" 20.

Apesar do arsenal propedêutico disponível, a freqüência de casos de PA sem causa aparente está em torno de $10 \%$ a $30 \%$ 7,19,39 e 25 a 50\% apresentarão recidiva nos 
dois anos subseqüentes ao primeiro episódio ${ }^{40}$. Pela tendência de recidiva, a PA sem causa aparente apresenta elevada taxa de morbidade e mortalidade ${ }^{4142}$.

É importante estabelecer o diagnóstico etiológico da PA, não só por sua implicação prognóstica ${ }^{6,18,20}$, mas para adotar o tratamento adequado ${ }^{20,43}$, melhorando a evolução do doente e reduzindo o risco de recidivas ${ }^{20,44}$.

Os estudos de Ranson ${ }^{45}$ e Miquel et al. ${ }^{46}$ demonstraram que aproximadamente $30 \%$ dos casos de PA inicialmente considerados sem causa aparente são, na realidade, secundários à microcálculos da vesícula biliar (MCV) não diagnosticados. Eles têm a tendência de migrar com facilidade pelo cístico ${ }^{47}$, encravar na papila duodenal ${ }^{9}$ e causar cólicas biliares, icterícia e PA ${ }^{18,48}$. Daí a importância do estudo ecoendoscópico nesses pacientes. Porém antes de entrarmos nessa questão alguns pontos devem ser definidos. Devemos em especial definir o que é microcálculo e o que são os microcristais biliares.

Definição de microcálculo e "barro biliar"

Algumas particularidades dos microcálculos, como radiotransparência ${ }^{10,49}$ e tamanho diminuto (dimensão máxima de $3 \mathrm{~mm})^{18,50}$, tornam-nos de difícil identificação pelo US, TC, CPER e CPRM ${ }^{51}$, métodos de imagem convencionais. Segundo esses autores, cálculos com essas características constituiriam microlitíase (Figuras 3 e 4).

O "barro biliar" caracteriza-se ao US ou EE como múltiplos sinais hiperecóicos, móveis, sem sombra acústica, que formam níveis no interior da vesícula biliar ${ }^{52,53}$ e o consideramos como microlitíase (Figuras 5 e 6).

\section{Gênese dos episódios de PA em doentes com microcálculos}

$O$ risco de $\mathrm{PA}$, nesses doentes relaciona-se ao tamanho dos cálculos, ao calibre do ducto cístico e ao calibre e comprimento do esfíncter de Oddi. Armstrong et al. ${ }^{17}$ demonstraram, em estudo prospectivo, que o diâmetro do ducto cístico era de 4,94 + 2,3 mm em doentes com PA e menor naqueles sem PA (grupo-controle). Além disso, revelaram que, em 64,5\% dos doentes com MCV (cálculos menores ou iguais a $3 \mathrm{~mm}$ ), a passagem pelo ducto cístico fora fácil, enquanto nos do grupo-controle em apenas 22,4\% ocorreu à passagem dos MCV devido ao menor diâmetro do ducto cístico. Fator mecânico importante é o diâmetro do esfíncter de Oddi, que varia de $2+1 \mathrm{~mm}$ constituindo obstáculo à passagem do cálculo, levando ou não à $P A^{54}$.

O comprimento do canal comum parece estar envolvido na gênese de surtos de PA, sendo muito maior nestes doentes que naqueles sem a doença ${ }^{17}$. Partindo-se dessa premissa, infere-se a importância dessa condição anatômica que aumenta o tempo de migração dos microcálculos ${ }^{17,54}$. Tais conceitos respaldam fortemente a teoria de que doentes com PA apresentam condições anatômicas que facilitam a migração de cálculos da vesícula biliar para o colédoco e cuja expulsão é mais demorada devido à maior distância a ser percorrida até o duodeno ${ }^{17,54}$. Outro autor demonstrou que, além do ducto cístico ser mais largo, o número de cálculos é maior em doentes com PA ${ }^{15}$. O que tem sido comprovado em nossa experiência onde todos os doentes com microlitíase apresentam múltiplos cálculos (Figura 3) ${ }^{55}$.

\section{Coleta e análise microscópica da bile}

Meltzer ${ }^{56}$ e Lyon ${ }^{57}$ descreveram quase simultaneamente a técnica de tubagem duodenal, de coleta e análise microscópica da bile, para a identificação de cristais de colesterol e de bilirrubinato de cálcio. Relegada ao esquecimento por muitos anos, vários autores a utilizaram na tentativa de reduzir o número de doentes sem diagnóstico etiológico da PA $32,41,42,58-62$.

Dentre as modalidades técnicas, para a análise da bile, podemos destacar o recolhimento com ou sem es-

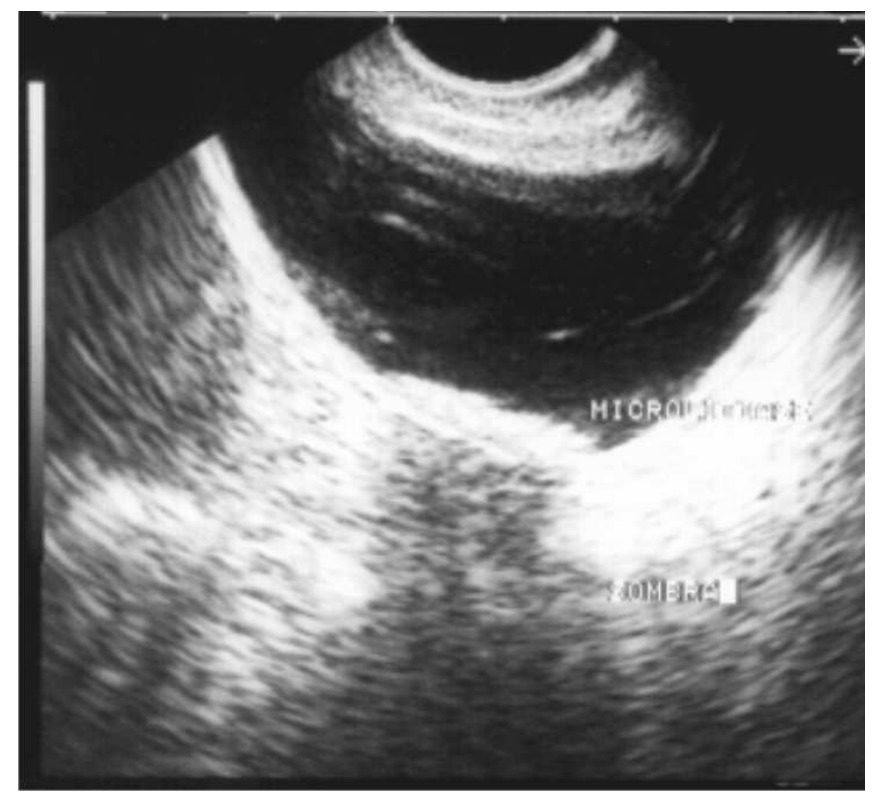

Figura 3 - EE com transdutor setorial eletrônico. Área hiperecóica formando nível com sombra acústica no corpo da vesícula biliar em paciente com vários episódios de PA.

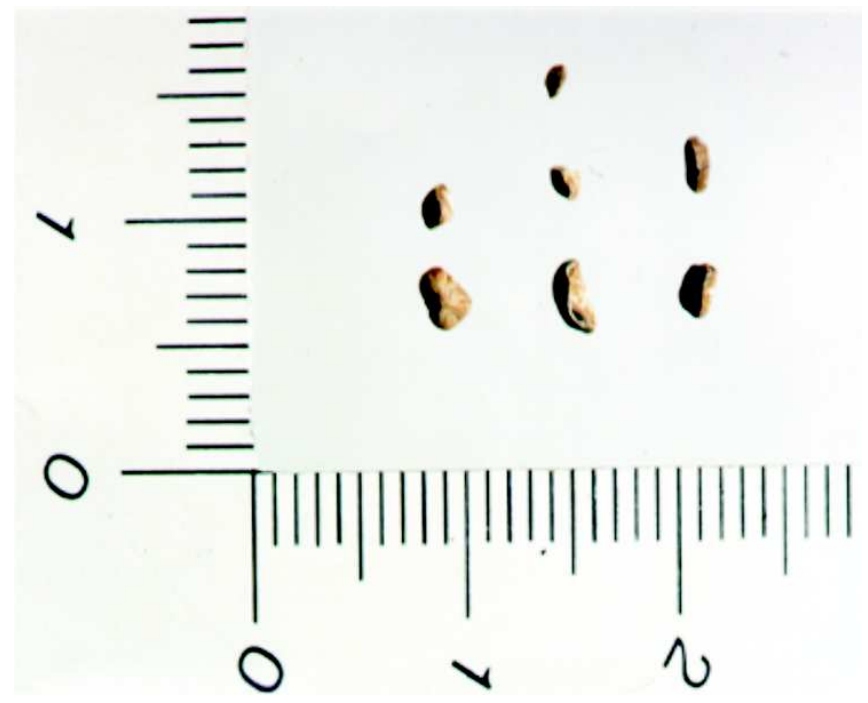

Figura 4 - Imagem de pequenos cálculos (menores que $3 \mathrm{~mm}$ ), causadores de episódios de PA Cálculos da peça cirúrgica até $3 \mathrm{~mm}$, inclusive. 



Figura 5 - Imagens ecoendoscópicas (a e b) de múltiplos sinais hiperecóicos, móveis, sem sombra acústica, que formam níveis no interior da vesícula biliar.

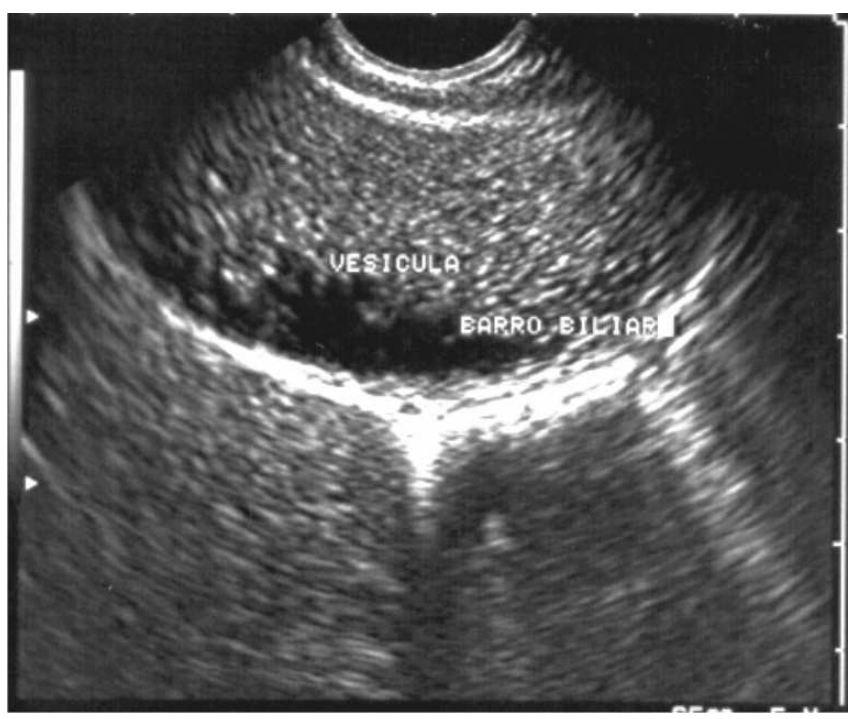

Figura 6 - EE com transdutor mecânico setorial. Espessamento da parede (colecistite aguda) e múltiplos sinais hiperecóicos móveis, sem sombra acústica, formando nível no interior da vesícula biliar ("barro biliar").

tímulo por colecistocinéticos, seja pelo posicionamento da sonda por fluoroscopia na segunda ou terceira porção duodenal ${ }^{41,42,63}$, pela coleta através do canal de aspiração do gastroscópio ${ }^{58}$; pela punção direta da vesícula biliar guiada por US ${ }^{64}$, por ecoendoscopia ${ }^{65}$ ou ainda pela coleta durante a CPER ${ }^{66}$.

\section{Correlação entre a presença de} microcristais e microcálculos

A análise microscópica da bile tem ganhado adeptos. Nos trabalhos realizados em doentes com PA sem causa aparente, demonstrou-se a presença de cristais de colesterol e de bilirrubinato de cálcio na bile coletada de vários deles. Submetidos à colecistectomia, encontraramse microcálculos não detectados por exames convencionais de imagem ${ }^{4261}$.

Há nítida correlação entre o encontro de cristais de colesterol e de bilirrubinato de cálcio na bile de doentes com litíase biliar conhecida ${ }^{67,68}$ e em doentes com diagnóstico de PA sem causa aparente ${ }^{42}$, nos quais a colecistectomia demonstrou a presença de microcálculos ${ }^{41}$

Sigman et al. ${ }^{63}$ e Chebli et al. ${ }^{66}$ não detectaram a presença de litíase em doentes com cristais na bile, mas encontraram vesículas com sinais de colecistite crônica ou colesterolose, e os doentes operados apresentaram melhora clínica de 50 a $100 \%$. Será que a colesterolose também pode causar PA? Essa é uma questão que tem nos intrigado, pois vários pacientes de nossa casuística com PA sem causa aparente ao serem submetidos a colecistectomia apresentam ao anatomopatológico colesterolose (Figuras 7,8 e 9)

\section{Valor da presença de microcristais na bile nos casos de pancreatite aguda}

A não identificação precisa do fator etiológico do surto de PA, favorece a ocorrência de novos episódios, às vezes fatais, em $33 \%$ a $48 \%$ dos doentes, antes da segunda admissão hospitalar2,69,70. É fácil perceber a importância de se estabelecer com precisão a etiologia do surto de PA, durante a hospitalização.

Neoptolemos et al. ${ }^{59}$ encontraram microcálculos em $60 \%$ dos doentes com e em $11 \%$ sem cristais de colesterol e de bilirrubinato ao exame da bile. A tamisação das fezes destes últimos pode aumentar a taxa de detecção de cálculos $^{15}$. Esse método não é prático nem fácil de ser realizado e, segundo Houssin et al. ${ }^{18}$, não é sensível bastante para identificar cálculos até $3 \mathrm{~mm}$, inclusive.

Outro problema é o "barro biliar", por muitos não aceito como fator etiológico da PA, o que pode induzir ao retardo no diagnóstico e, conseqüentemente, impedir o pronto tratamento dos doentes ${ }^{42,53,71}$. Lee et al. ${ }^{42}$ demonstraram que a ocorrência de barro biliar parece ser maior quando um paciente sofre de ataques recorrentes de PA e que essa causa é subestimada em pacientes com PA sem causa aparente. Inclusive a colecistectomia profilática, tem sido realizada quando a suspeita diagnóstica é forte, para evitar a possibilidade de novos episódios ${ }^{72}$. 



Figura 7 - Imagens ecoendoscópicas de pacientes com PA sem causa aparente. Em (a) observe a presença de múltiplos ecos hiperecóicos, onde alguns deles apresentam sombra acústica. O histológico da peça revelou colesterolose. Em (b) imagem da vesícula biliar com líquido perivesicular. Durante a operação observou-se microlitíase não detectada pela EE e o anátomo-patológico mostrou colesterolose.

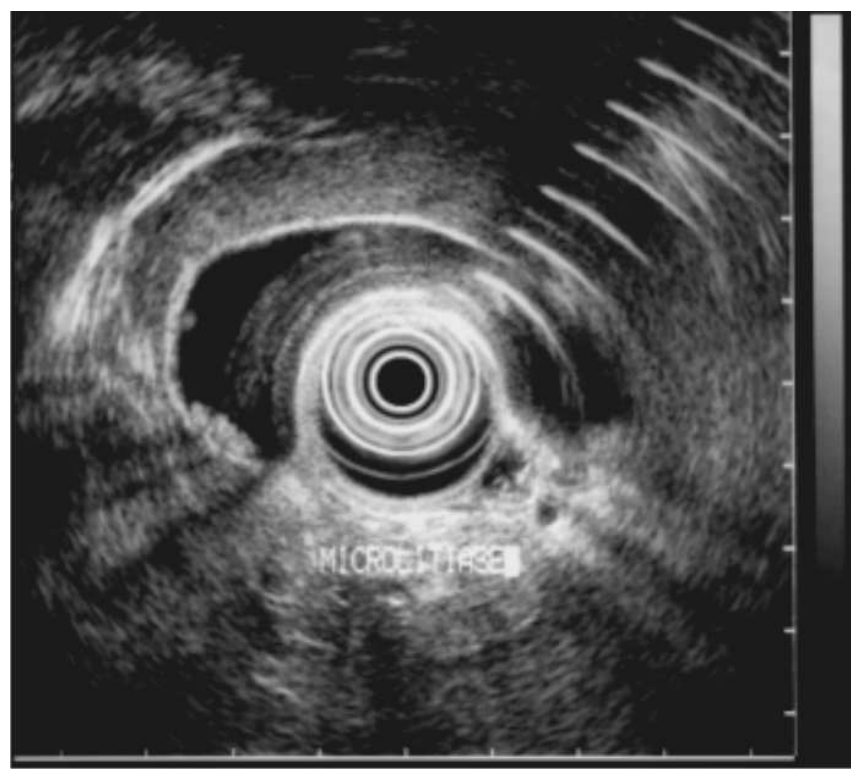

Figura 8 - EE radial. Focos hiperecóicos com sombra acústica no fundo da vesícula biliar e uma diminuta elevação hiperecóica sem sombra acústica aderida à parede de seu corpo.

\section{Diagnóstico da etiologia biliar Diagnóstico da coledocolitíase}

Sugiyama et al. ${ }^{23}$ demonstraram sensibilidade da EE no diagnóstico da coledocolitíase de $100 \%$ contra $43 \%$ do US e $57 \%$ da TC. A sensibilidade do US para o diagnóstico de colelitíase varia entre 87 e $98 \%{ }^{26}$ e para coledocolitíase de 22 e $75 \%{ }^{33}$. A tabela 1 mostra os resultados de vários trabalhos publicados na literatura onde são avaliados os exames de US, TC e EE para o diagnóstico da coledocolitíase.

Os autores estudaram 22 pacientes com suspeita de microcoledocolitíase durante surtos de PA, observaram que a EE identificou a presença de cálculo em $86,7 \%$ dos casos (tamanho médio do cálculo: $5 \mathrm{~mm}$ ), sensibilidade superior à da CPER (53,3\%). O colédoco foi bem estudado em

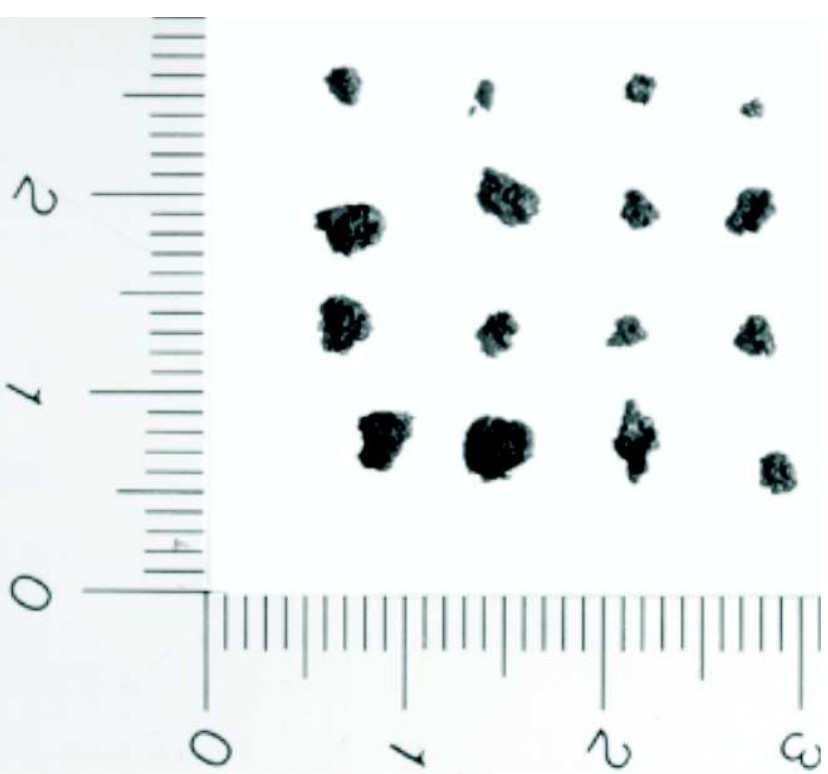

Figura 9 - Microcálculos encontrados na peça operatória.

todos os pacientes, não sendo observadas complicações relacionadas ao procedimento. Concluíram que a EE é capaz de identificar cálculos pequenos e dilatações biliares, devendo ser considerada como uma alternativa diagnóstica nos pacientes com suspeita de litíase coledoceana ou nos casos de insucesso da CPER (Figura 10) ${ }^{73}$.

\section{Diagnóstico da microlitíase}

Após investigação diagnóstica sistemática (clínica, bioquímica e por métodos de imagem) para a identificação do fator etiológico da PA, cerca de 10\% a 30\% dos doentes não apresentam causa definida e são rotulados como PA sem causa aparente ${ }^{75}$. É no mínimo controversa a afirmação de que caso a etiologia de um episódio de PA permaneça obscura, depois de investigação endoscópica pormenorizada (CPER ou CPER + coleta de bile), deve ser chamada "idiopática"20,39. 
Tabela 1 - Resultados dos diversos métodos de imagem no diagnóstico da coledocolitíase.

\begin{tabular}{|c|c|c|c|c|c|c|c|}
\hline Autor, ano & $\mathrm{N}$ & Método & Sens \% & Esp \% & $V P+\%$ & VP- \% & Acur \% \\
\hline \multirow[t]{3}{*}{ Amouyal, $1994{ }^{33}$} & 62 & $\cdot$ US & 25 & 100 & 100 & 56 & \\
\hline & & $\cdot \mathrm{TC}$ & 75 & 94 & 92 & 78 & \\
\hline & & $\cdot \mathrm{EE}$ & 97 & 100 & 100 & 97 & \\
\hline \multirow[t]{3}{*}{ Sugiyama, $1995^{23}$} & 23 & $\cdot$ US & 43 & & & & \\
\hline & & $\cdot \mathrm{TC}$ & 57 & & & & \\
\hline & & $\cdot \mathrm{EE}$ & 100 & 100 & & & 100 \\
\hline \multirow[t]{3}{*}{ Sugiyama, 1997 74* } & 142 & $\cdot$ US & 63 & 95 & & & 83 \\
\hline & & $\cdot \mathrm{TC}$ & 71 & 97 & & & 87 \\
\hline & & $\cdot \mathrm{EE}$ & 96 & 100 & & & 99 \\
\hline \multirow[t]{3}{*}{ Sugiyama, $1998{ }^{34}$} & 35 & US & 47 & 90 & & & 71 \\
\hline & & $\cdot \mathrm{TC}$ & 47 & 95 & & & 74 \\
\hline & & $\cdot \mathrm{EE}$ & 100 & 100 & & & 100 \\
\hline \multirow[t]{3}{*}{ Chak, 199925} & 36 & $\cdot$ US & 50 & 100 & 100 & 74 & 83 \\
\hline & & - CPER & 92 & 87 & 79 & 94 & 89 \\
\hline & & $\cdot \mathrm{EE}$ & 91 & 100 & 100 & 95 & 97 \\
\hline \multirow[t]{2}{*}{ Ardengh, 2000 73** } & 22 & - CPER & 53,3 & 100 & 100 & 50 & 68,2 \\
\hline & & $\cdot \mathrm{EE}$ & 86,7 & 80 & 92,9 & 66,7 & 77,3 \\
\hline \multirow[t]{3}{*}{ Liu, 200127} & 100 & US & 26 & 100 & & & 75 \\
\hline & & - CPER & 97 & 95 & & & 96 \\
\hline & & $\cdot \mathrm{EE}$ & 97 & 98 & & & 98 \\
\hline
\end{tabular}

*Pacientes com ou sem história de PA.

** Microcoledocolitíase.

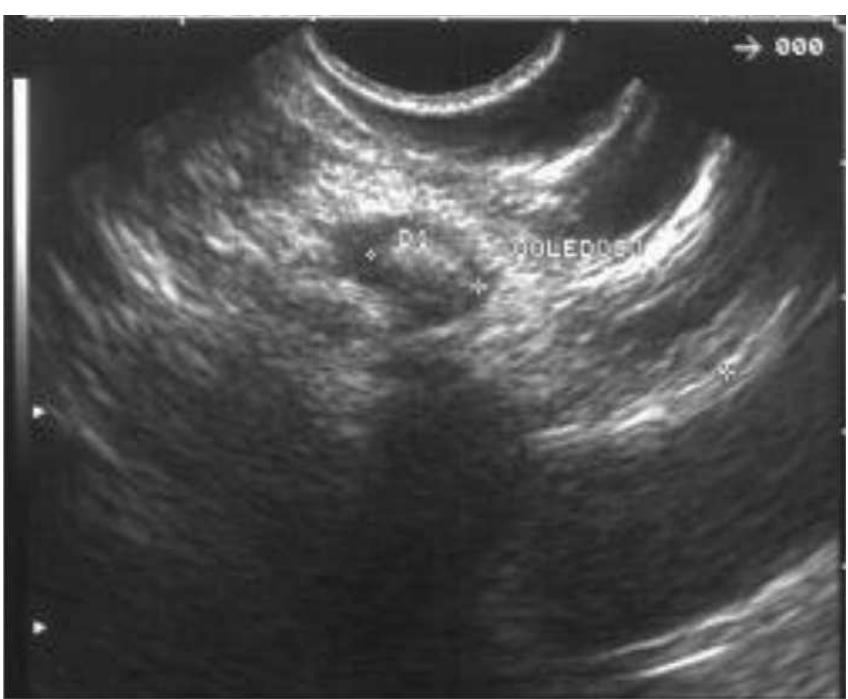

Figura 10 - Imagem ecoendoscópica de área hiperecóica arciforme com sombra acústica no interior do colédoco em paciente com PA sem causa aparente. Essa imagem foi confirmada pela CPER.

A identificação de doentes com microlitíase desperta grande interesse devido à possibilidade de ocorrerem surtos recidivantes de PA e por permitir terapêutica precoce, principalmente quando a doença é silenciosa ou pouco sintomática ${ }^{18,59}$. Por essa razão, a introdução de métodos diagnósticos sensíveis, pouco invasivos e o ressurgimento de métodos antigos (coleta de bile para a pesquisa de cristais) tornaram-se atraentes, além de métodos mais invasivos como a manometria endoscópica ${ }^{76}$.

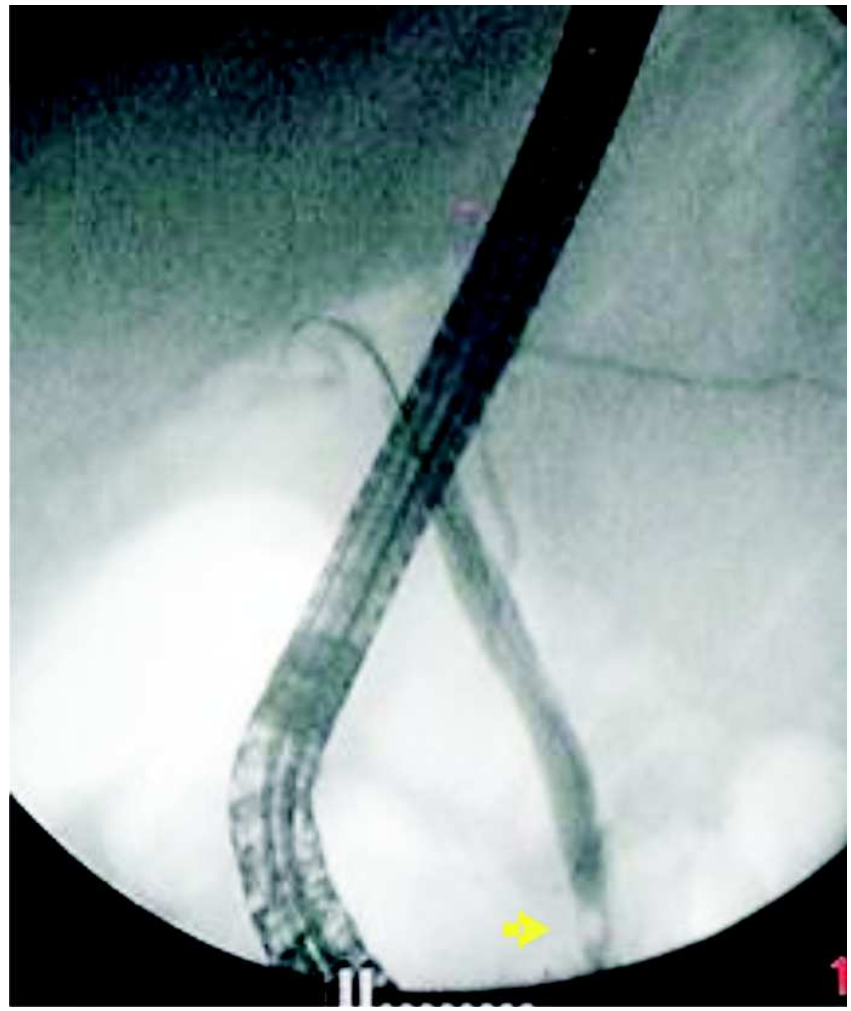

Figura 11 - Imagem de CPER demonstrando microcálculo (seta preta) no interior de via biliar sem dilatação.

Acerca desse ponto de vista, parecem avultar em importância as técnicas endoscópicas avançadas: a CPER ${ }^{77}$, a CPER com coleta de bile, para a pesquisa de 
microcristais ${ }^{66,68}$ e a EE com o objetivo de detectar a microlitíase $55,73,78$. Assim o diagnóstico de cálculos menores que $3 \mathrm{~mm}$, (microcálculos) é de fundamental importância para a adoção de medida terapêutica adequada.

A demonstração radiológica da litíase biliar depende da diferença de tonalidade entre o cálculo e o meio que o envolve ${ }^{10,79}$. Os doentes com cálculos radiotransparentes e migrantes apresentam episódios de cólicas biliares, PA recidivante e icterícia transitória ${ }^{10}$, apesar do aspecto radiológico normal das vias biliares a CPRM, CPER, e US. Consideramos o "barro biliar" (figura 4) como microlitíase, pois o "barro" e cálculos com diâmetro maior que $4 \mathrm{~mm}$, por apresentarem sombra acústica, podem ser identificados pelo US, ${ }^{80}$, sendo confundidos "in vivo" segundo Simeone et al. ${ }^{81}$ e diferençados "in vitro" conforme Filly et $a{ }^{52}$.

Simeone et al. ${ }^{81}$ demonstraram a presença de microcálculos em 8,3\% dos doentes com "barro biliar" submetidos ao tratamento cirúrgico. Filly et al. ${ }^{52}$ concluíram que a presença de "barro biliar" deve ser considerada anormal, pois pode haver precipitados de bilirrubinato de cálcio ou colesterol (microcálculos).

A possibilidade de encontrar MCV em doente com litíase constatada por métodos de imagem, respeitando-se o conceito de tamanho menor ou igual a $3 \mathrm{~mm}$ de diâmetro, está em torno de $25 \%{ }^{18}$. Esta cifra aumenta para $40 \%$ quando o cálculo passa de $3 \mathrm{~mm}$ para $5 \mathrm{~mm}^{82,83} \mathrm{e}$, se utilizado o critério da radiotransparência, cai para $10 \%{ }^{83}$.

Doentes com PA recidivante que apresentam US sem alterações podem exibir cálculos durante o ato operatório $^{84,85}$. O tamanho e a densidade dos cálculos são os fatores mais importantes na ocorrência desses falsos resulta$\operatorname{dos}^{85}$.

A CPER é empregada no diagnóstico etiológico de doentes com PA sem causa aparente, identificando-se condições potencialmente tratáveis em 30\% a 70\% ${ }^{76}$ dos casos e cálculos (Figura 11) entre $4 \%^{76}$ a $15 \%{ }^{19,86}$. Mas é preciso ter em mente que esse exame é capaz de elevar os níveis de amilase em 30\% a $75 \%$ dos doentes assintomáticos $^{87}$ e a PA é a mais freqüente complicação após o exame, variando as cifras de $0,5 \%$ a $17 \%{ }^{76,87}$. Thoeni et al. ${ }^{88}$ relataram sinais tomográficos de PA de leve a grave em $28 \%$ dos doentes submetidos a CPER diagnóstica.

\section{Análise Crítica da Literatura}

Dahan et al. ${ }^{24}$, em estudo prospectivo, compararam a acurácia da EE e da coleta de bile para a pesquisa de cristais biliares, em doentes com suspeita da etiologia biliar para os seus sintomas (PA, icterícia transitória e cólicas no hipocôndrio direito) e com diagnóstico negativo de cálculos biliares com o US. O estudo incluiu 45 doentes, 24 com colecistolitíase confirmada durante o ato operatório. A sensibilidade, a especificidade, os valores preditivos positivo e negativo para a EE foram, respectivamente, $96 \%$, $86 \%, 89 \%$ e $95 \%$ e para a coleta de bile $67 \%, 91 \%, 89 \%$ e $70 \%$. O maior problema da EE foi à existência de três falsos positivos. Na pesquisa desse autor a obtenção de imagens tidas como cálculos deveu-se à reverberação acús- tica pelo movimento da parede da vesícula, formando artefatos confundidos com microcálculos ${ }^{24,55}$.

Em nosso estudo sobre 36 doentes com PA sem causa aparente a sensibilidade foi de $92,6 \%$ e valor preditivo positivo de 86,2\%, na identificação dos MCV. Com base nessas estimativas, infere-se que a EE é segura e oferece boa precisão diagnóstica ${ }^{55}$ e que os prováveis fatores responsáveis, em maior ou menor grau, por essa alta taxa de detecção de microlitíase incluem: a vesícula biliar intimamente relacionada ao estômago e à parede duodenal; a distância reduzida $(0,5 \mathrm{~mm})$ entre o transdutor ecoendoscópico e a vesícula biliar comparativamente à do transdutor abdominal durante a realização do US, a fácil obtenção do ponto focal ideal para conseguir imagens sem distorções; o estudo de toda a vesícula biliar (infundíbulo, corpo, fundo) e do ducto cístico, mesmo em doentes com quadros graves de PA e a utilização de freqüências de 5,0, 7,5 e $12 \mathrm{MHz}$, intensificando os pormenores das imagens.

Por outro lado, a especificidade e o valor preditivo negativo foram $55,6 \%$ e $71,4 \%$, respectivamente. Essas estimativas são questionáveis e pouco precisas ${ }^{55}$. Os principais fatores responsáveis, em maior ou menor grau, por estas cifras são: a amostra muito pequena de indivíduos sem a doença e a grande probabilidade de os doentes examinados serem portadores da etiologia biliar como causa dos episódios de PA.

Em um estudo envolvendo 168 pacientes com PA sem causa aparente, Frossard et al. ${ }^{78}$ conseguiram identificar com a EE a presença de litíase biliar em 103 (61\%) (colelitíase ou microlitíase em 52, barro biliar em 12, coledocolitíase em 10, mais de um destes achados em 29). Afastadas outras causas (pancreatite crônica, tumores e etc.), apenas 37 permaneceram com diagnóstico de entrada inalterado.

Estudo semelhante empregando a EE em 44 pacientes com PA idiopática, Norton \& Alderson ${ }^{26}$ encontraram causa definida para a PA em 18 pacientes $(41 \%)$ e uma provável etiologia em 14 (31,8\%). Apenas nove permaneceram sem esclarecimento diagnóstico. A EE apresentou 2 resultados falso-positivos e não foi possível sua realização por problemas técnicos em um paciente.

Liu et al. ${ }^{89}$ estudaram prospectivamente 89 pacientes com PA sem causa aparente. Todos foram submetidos ao US e TC, até 24 horas da admissão hospitalar e a uma CPER. Após esses exames 18 pacientes foram rotulados como portadores de PA sem causa aparente. Todos foram submetidos à EE. Ela revelou microcálculos vesiculares em $14(77,7 \%)$ e em três havia concomitância com cálculos no interior do colédoco (21,4\%). Esses achados demonstram que um paciente com PA não deve ser rotulado como sem causa aparente antes da realização de uma EE.

Tandon \& Topazian $^{90}$ avaliaram o papel da EE no diagnóstico da causa de episódios de PA sem causa aparente. Em 21/31 pacientes (68\%) a EE revelou o fator etiológico. A etiologia biliar foi encontrada em 14\%, pancreatite crônica inicial em 45\%, pâncreas divisum em $6,5 \%$ e tumores em 3,2\%. Em 32\% dos casos a EE foi normal e não conseguiu identificar a causa. Os resultados 
desse estudo mostram que a EE é menos invasiva que a CPER, demonstrando de forma efetiva a etiologia de episódios de PA em mais de 2/3 dos casos.

Em pacientes com PA biliar, a EE realizada antes da CPER auxilia na seleção dos pacientes com necessidade de terapêutica endoscópica, reduzindo a necessidade de colangiografia diagnóstica (e seus riscos potenciais) em cerca de $50 \%$ deles 27,34 .

Levy et al. ${ }^{91}$ em trabalho multicêntrico avaliaram múltiplos fatores para prever a etiologia biliar de um episódio de PA. Foram estudados 213 pacientes com PA, onde a causa foi biliar em $62 \%$, alcoólica em $35 \%$ e outras causas em 13\%. Em 15\% dos pacientes com etiologia biliar apenas a EE fez o diagnóstico, pois os outros exames foram negativos. Os autores concluem que quando uma EE é realizada para confirmar ou excluir a presença de cálculos, como origem da PA, a idade, o sexo e a alanina transaminase obtida na admissão são os únicos fatores preditivos que falam a favor da origem biliar.

Destarte Shimpi et al. ${ }^{76}$ determinaram a acurácia diagnóstica da EE e da CPER em pacientes com PAI. A EE revelou o diagnóstico em 44\% (28/63) dos pacientes, enquanto a CPER fez o diagnóstico em 71\% (45/63). A taxa de ocorrência de PA após a CPER e a manometria foi de $17 \%$. Não houve nenhuma complicação relacionada à $\mathrm{EE}$ nem a EE-PAAF que foi realizada em dois doentes. Os autores concluem que a EE tem um importante papel no diagnóstico da causa da PA sem causa aparente.

\section{CONSIDERAÇÕES FINAIS}

Em uma porcentagem grande de pacientes com episódios de pancreatite aguda, a etiologia pode ser estabelecida com base na história inicial, exame físico, exames laboratoriais, bioquímico e com o auxílio de um simples ultra-som abdominal. Por outro lado quando esta sequência racional de conduta não identifica a causa do episódio uma parcela de pacientes são considerados como sem causa aparente. Essa situação é preocupante, pois esses episódios de pancreatite aguda tendem a recorrência com altas taxas de morbidade de mortalidade. Entretanto a causa e mais frequentemente o tratamento destes pacientes podem prevenir ataques recorrentes de pancreatite. Destarte o uso de todas as ferramentas disponíveis para o diagnóstico preciso, deve ser utilizado de forma agressiva para se detectar, por exemplo, casos de microlitíase ou cálculos ocultos, não devendo esquecer-se das anormalidades dos ductos biliares e pancreáticos e da disfunção do esfíncter de Oddi.

\title{
A B S T R A C T
}

\begin{abstract}
In a large number of patients, with episodes of acute pancreatitis the etiology is not identified, even after initial clinical history detailed physical examination, laboratory tests and biochemical exams and an transabdominal ultrasound. This patient are considered with a unexplained acute pancreatitis. In this cases the treatment is restricted to improvement of symptoms. These patients after treated tend to have new episodes with the risk of raising the rates of morbidity and mortality. Therefore, the identification of a cause and its prompt treatment prevent at recurrent episodes of pancreatitis. This review aims to draw attention to how best diagnostic approach when the light of evidence-based medicine, to search for causes of difficult identification with microlithiasis, occult stones, the anatomical variations of biliary and pancreatic duct and in addition to sphincter of Oddi dysfunction.
\end{abstract}

Key words: Gallstones. Endosonography. Pancreatitis. Diagnosis.

\section{REFERÊNCIAS}

1. Mehdi M, Deutsch JP, Arrive L, Ayadi K, Ladeb MF, Tubiana JM. [Acute pancreatitis]. Ann Radiol. (Paris). 1996; 39(1):37-44.

2. Pandol SJ. Acute pancreatitis. Curr Opin Gastroenterol. 2006; 22(5):481-6

3. Dalzell DP, Scharling ES, Ott DJ, Wolfman NT. Acute pancreatitis: the role of diagnostic imaging. Crit Rev Diagn Imaging. 1998; 39(5):339-63.

4. Appelros S, Borgstrom A. Incidence, aetiology and mortality rate of acute pancreatitis over 10 years in a defined urban population in Sweden. Br J Surg. 1999; 86(4):465-70.

5. Bradley $\mathrm{EL}, 3^{\text {rd }}$, Allen K. A prospective longitudinal study of observation versus surgical intervention in the management of necrotizing pancreatitis. Am J Surg. 1991; 161(1):19-24; discussion 24-5.

6. Levy MJ, Geenen JE. Idiopathic acute recurrent pancreatitis. Am J Gastroenterol. 2001; 96(9):2540-55

7. Marshall JB. Acute pancreatitis. A review with an emphasis on new developments. Arch Intern Med. 1993; 153(10):1185-98.

8. Patti MG, Pellegrini CA. Gallstone pancreatitis. Surg Clin North Am. 1990; 70(6):1277-95.
9. Acosta JM, Ledesma CL. Gallstone migration as a cause of acute pancreatitis. N Engl J Med. 1974; 290(9):484-7.

10. Dayan L, Cherif-Zahar K, Lepage B, Bories-Azeau A. [Diagnostic traps and procedure to follow in radio-invisible biliary calculi]. J Chir (Paris). 1976; 111(4):431-42

11. Goodman AJ, Neoptolemos JP, Carr-Locke DL, Finlay DB, Fossard DP. Detection of gall stones after acute pancreatitis. Gut. 1985; 26(2):125-32

12. Lee SP, Nicholls JF. Nature and composition of biliary sludge. Gastroenterology. 1986; 90(3):677-86.

13. Aube C, Delorme B, Yzet T, Burtin P, Lebigot J, Pessaux P et al. MR cholangiopancreatography versus endoscopic sonography in suspected common bile duct lithiasis: a prospective, comparative study. AJR Am J Roentgenol. 2005; 184(1):55-62

14. Moon JH, Cho YD, Cha SW, Cheon YK, Ahn HC, Kim YS et al. The detection of bile duct stones in suspected biliary pancreatitis: comparison of MRCP, ERCP, and intraductal US. Am J Gastroenterol. 2005; 100(5):1051-7.

15. McMahon MJ, Shefta JR. Physical characteristics of gallstones and the calibre of the cystic duct in patients with acute pancreatitis. $\mathrm{Br}$ J Surg. 1980; 67(1):6-9.

16. De Bolla AR, Obeid ML. Mortality in acute pancreatitis. Ann R Coll Surg Engl. 1984; 66(3):184-6. 
17. Armstrong CP, Taylor TV, Jeacock J, Lucas S. The biliary tract in patients with acute gallstone pancreatitis. Br J Surg. 1985; 72(7):551-5.

18. Houssin D, Castaing D, Lemoine J, Bismuth $H$. Microlithiasis of the gallbladder. Surg Gynecol Obstet. 1983; 157(1):20-4.

19. Grendell JH. Idiopathic acute pancreatitis. Gastroenterol Clin North Am. 1990; 19(4):843-8.

20. Tarnasky PR, Hawes RH. Endoscopic diagnosis and therapy of unexplained (idiopathic) acute pancreatitis. Gastrointest Endosc Clin N Am. 1998;8(1):13-37

21. Pezzilli R, Billi P, Miglioli M, Gullo L. Serum amylase and lipase concentrations and lipase/amylase ratio in assessment of etiology and severity of acute pancreatitis. Dig Dis Sci. 1993;38(7):1265-9.

22. United Kingdom guidelines for the management of acute pancreatitis. British Society of Gastroenterology. Gut. 1998; 42 Suppl 2:S1-13

23. Sugiyama M, Wada N, Atomi $Y$, Kuroda A, Muto T. Diagnosis of acute pancreatitis: value of endoscopic sonography. AJR Am J Roentgenol. 1995; 165(4):867-72.

24. Dahan P, Andant C, Levy P, Amouyal P, Amouyal G, Dumont M et al. Prospective evaluation of endoscopic ultrasonography and microscopic examination of duodenal bile in the diagnosis of cholecystolithiasis in 45 patients with normal conventional ultrasonography. Gut. 1996; 38(2):277-81.

25. Chak A, Hawes RH, Cooper GS, Hoffman B, Catalano MF, Wong $\mathrm{RC}$ et al. Prospective assessment of the utility of EUS in the evaluation of gallstone pancreatitis. Gastrointest Endosc. 1999; 49(5):599604.

26. Norton SA, Alderson D. Endoscopic ultrasonography in the evaluation of idiopathic acute pancreatitis. Br J Surg. 2000; 87(12):1650-5

27. Liu CL, Lo CM, Chan JK, Poon RT, Lam CM, Fan ST et al. Detection of choledocholithiasis by EUS in acute pancreatitis: a prospective evaluation in 100 consecutive patients. Gastrointest Endosc. 2001; 54(3):325-30.

28. Mitchell RM, Byrne MF, Baillie J. Pancreatitis. Lancet. 2003; 361(9367):1447-55

29. Scholmerich J, Johannesson T, Brobmann G, Wimmer B, Thiedemann B, Gross $V$ et al. [Sonography in acute pancreatitisdiagnosis, assessment of etiology and evaluating prognosis]. Ultraschall Med. 1989; 10(6):290-4.

30. Bolognese A, Muttillo IA, Scopinaro F, Banci M, Amadori LM, De Martino $\mathrm{F}$ et al. [Biliary scintigraphy vs. ultrasonography in the etiological diagnosis of acute pancreatitis]. J Chir (Paris). 1996; 133(2):78-81.

31. Parodi HC, Gutierrez S, Lattanzi M, Martinez R, Colombato LO. [Value of laboratory tests and echography in the diagnosis of biliary disease in the initial phase of acute pancreatitis]. Acta Gastroenterol Latinoam. 1990; 20(3):137-44

32. Bel FJ, Aparisi L, Garcia-Tell G, Rosello JV, Rodrigo JM. [Biliary drainage in the diagnosis of microlithiasis. Value in acute idiopathic pancreatitis and in persistent pain in the right hypochondrium]. Rev Esp Enferm Dig. 1994; 85(5):343-7.

33. Amouyal $P$, Amouyal $G$, Levy $P$, Tuzet $S$, Palazzo L, Vilgrain $V$ et al. Diagnosis of choledocholithiasis by endoscopic ultrasonography. Gastroenterology. 1994; 106(4):1062-7.

34. Sugiyama M, Atomi $Y$. Acute biliary pancreatitis: the roles of endoscopic ultrasonography and endoscopic retrograde cholangiopancreatography. Surgery. 1998; 124(1):14-21.

35. Balthazar EJ, Robinson DL, Megibow AJ, Ranson JH. Acute pancreatitis: value of CT in establishing prognosis. Radiology. 1990; 174(2):331-6.

36. Norton SA, Alderson D. Prospective comparison of endoscopic ultrasonography and endoscopic retrograde cholangiopancreatography in the detection of bile duct stones. Br J Surg. 1997; 84(10):1366-9.

37. Barthet M. [Diagnosis of biliary origin of acute pancreatitis]. Gastroenterol Clin Biol. 2001; 25(1 Suppl):1S12-7.

38. Sica GT, Braver J, Cooney MJ, Miller FH, Chai JL, Adams DF. Comparison of endoscopic retrograde cholangiopancreatography with MR cholangiopancreatography in patients with pancreatitis. Radiology. 1999; 210(3):605-10.

39. Steinberg WM. Acute pancreatitis - never leave a stone unturned. N Engl J Med. 1992; 326(9):635-7.
40. Khalid A, Slivka A. Approach to idiopathic recurrent pancreatitis. Gastrointest Endosc Clin N Am. 2003;13(4):695-716, x

41. Ros E, Navarro S, Bru C, Garcia-Puges A, Valderrama R. Occult microlithiasis in 'idiopathic' acute pancreatitis: prevention of relapses by cholecystectomy or ursodeoxycholic acid therapy. Gastroenterology. 1991; 101(6):1701-9.

42. Lee SP, Nicholls JF, Park HZ. Biliary sludge as a cause of acute pancreatitis. N Engl J Med. 1992; 326(9):589-93.

43. Neoptolemos JP, Carr-Locke DL, London NJ, Bailey IA, James D, Fossard DP. Controlled trial of urgent endoscopic retrograde cholangiopancreatography and endoscopic sphincterotomy versus conservative treatment for acute pancreatitis due to gallstones. Lancet. 1988; 2(8618):979-83.

44. Potts JR, $3^{\text {rd. }}$ Acute pancreatitis. Surg Clin North Am. 1988; 68(2):281-99.

45. Ranson JH. Acute pancreatitis: pathogenesis, outcome and treatment. Clin Gastroenterol. 1984; 13(3):843-63.

46. Miquel JF, Rollan A, Guzman S, Nervi F. Microlithiasis and cholesterolosis in 'idiopathic' acute pancreatitis. Gastroenterology. 1992; 102(6):2188-90.

47. Seror J, Rives J, Stoppa R. [Importance of the concept of microlithiasis in biliary pathology; sphincteral and choledochal incidences]. Afr Francaise Chir. 1961; 19:166-9.

48. Moskovitz M, Min TC, Gavaler JS. The microscopic examination of bile in patients with biliary pain and negative imaging tests. Am J Gastroenterol. 1986; 81(5):329-33.

49. Pfefferman R, Luttwak EM. Gallstone pancreatitis. Exploration of the biliary system in pancreatitis of undetermined origin. Arch Surg. 1971; 103(4):484-6.

50. Bertrand L, Lamarque JL. [Biliary microlithiasis. Medico-radiological observations]. Nouv Presse Med. 1975; 4(44):3135-8.

51. de Ledinghen V, Lecesne R, Raymond JM, Gense V, Amouretti M, Drouillard J et al. Diagnosis of choledocholithiasis: EUS or magnetic resonance cholangiography? A prospective controlled study. Gastrointest Endosc. 1999; 49(1):26-31.

52. Filly RA, Allen B, Minton MJ, Bernhoft R, Way LW. In vitro investigation of the origin of echoes with biliary sludge. J Clin Ultrasound. 1980; 8(3):193-200

53. Lee SP, Hayashi A, Kim YS. Biliary sludge: curiosity or culprit? Hepatology. 1994; 20(2):523-5.

54. Barraya L, Pujol Soler R, Yvergneaux JP. [The region of Oddi's sphincter: millimetric anatomy]. Presse Med. 1971; 79(55):2527-34.

55. Ardengh JC, Malheiros CA, Ganc AJ, Ferrari A. Endoscopic ultrasound (EUS) in the diagnosis of gallbladder microlithiasis in patients with idiopathic acute pancreatitis. Digestion. 1998; 59(3):40(136)

56. Meltzer SJ. The disturbance of the law of contrary innervation as a pathogenic factor in diseases of the bile ducts and the gallbladder. Am J Med Sci. 1917; 153:469.

57. Lyon BBV. Diagnosis and treatment of diseases of the gallbladder and biliary ducts, preliminary report an a new method. JAMA. 1919: 73:980-2

58. Negro P, Flati G, Flati D, Porowska B, Tuscano D, Carboni M. Occult gallbladder microlithiasis causing acute recurrent pancreatitis. A report of three cases. Acta Chir Scand. 1984; 150(6):503-6.

59. Neoptolemos JP, Davidson BR, Winder AF, Vallance D. Role of duodenal bile crystal analysis in the investigation of 'idiopathic' pancreatitis. Br J Surg. 1988; 75(5):450-3.

60. Humbert P, Casals A, Boix J, Planas R, Morillas R, Barranco C et al. [Usefulness of microscopic study of the duodenal bile in the diagnosis of pancreatitis of unknown cause]. Rev Esp Enferm Apar Dig. 1989: 75(5):471-4.

61. Reyes Lopez A, Mino Fugarolas G, Costan Rodero G, Perez Rodriguez E, Montero Alvarez JL, Cabrera D. [Value of duodenal drainage in the etiologic diagnosis of acute pancreatitis]. Rev Esp Enferm Dig. 1993; 83(5):363-6.

62. Herrera Ballester A, Canelles Gamir P, Orti Ortin E, Ortega Gonzalez E, Soler Ros JJ, Benages Martinez A. [Biliary drainage in the diagnosis of acute idiopathic pancreatitis]. Rev Esp Enferm Dig. 1994; 86(5):813-7

63. Sigman $\mathrm{HH}$, Goldberg N, Niloff $\mathrm{PH}$, Lachance $\mathrm{C}$. Use of biliary drainage in diagnosis of biliary tract disease. Am J Gastroenterol. 1977: 67(5):439-43. 
64. Hussaini SH, Kennedy C, Pereira SP, Wass JA, Dowling RH. Ultrasound-guided percutaneous fine needle puncture of the gallbladder for studies of bile composition. Br J Radiol. 1995; 68(807):271-6

65. Jacobson BC, Waxman I, Parmar K, Kauffman JM, Clarke GA, Van Dam J. Endoscopic ultrasound-guided gallbladder bile aspiration in idiopathic pancreatitis carries a significant risk of bile peritonitis. Pancreatology. 2002; 2(1):26-9.

66. Chebli JM, Martins Junior EV, Gaburri AK, Ferreira LE, Gil JZ, Neves MM. [Microcrystals and biliary sludge: pathogenesis and clinical significance]. Arq Gastroenterol. 1996; 33(4):232-43.

67. Abbas A, Baumann R, Schutz JF, Maillard D, Sondag D, Weill JP. [Cholesterol crystals and biliary lithiasis. Importance of the study of bile collected by duodenal intubation]. Gastroenterol Clin Biol. 1984; 8(5):454-7.

68. Buscail L, Escourrou J, Delvaux M, Guimbaud R, Nicolet T, Frexinos $J$ et al. Microscopic examination of bile directly collected during endoscopic cannulation of the papilla. Utility in patients with suspected microlithiasis. Dig Dis Sci. 1992; 37(1):116-20.

69. Paloyan D, Simonowitz D, Skinner DB. The timing of biliary tract operations in patients with pancreatitis associated with gallstones. Surg Gynecol Obstet. 1975; 141(5):737-9

70. Ranson $\mathrm{JH}$. The timing of biliary surgery in acute pancreatitis. Ann Surg. 1979; 189(5):654-63

71. Marotta PJ, Gregor JC, Taves DH. Biliary sludge: a risk factor for 'idiopathic' pancreatitis? Can J Gastroenterol. 1996; 10(6):385-8.

72. Venneman NG, Buskens E, Besselink MG, Stads S, Go PM, Bosscha $\mathrm{K}$ et al. Small gallstones are associated with increased risk of acute pancreatitis: potential benefits of prophylactic cholecystectomy? Am J Gastroenterol. 2005; 100(11):2540-50.

73. Ardengh JC, Ganc AJ, Ferrari A, Malheiros CA, Rahal F. Accuracy of endoscopic ultrasonography (EUS) for diagnosis of microcholedocholithiasis in patients with acute pancreatitis. Endoscopy. 2000; 32:A27(P68).

74. Sugiyama M, Atomi Y. Endoscopic ultrasonography for diagnosing choledocholithiasis: a prospective comparative study with ultrasonography and computed tomography. Gastrointest Endosc. 1997; 45(2):143-6.

75. Norton SA, Cheruvu CV, Collins J, Dix FP, Eyre-Brook IA. An assessment of clinical guidelines for the management of acute pancreatitis. Ann R Coll Surg Engl. 2001; 83(6):399-405.

76. Shimpi RA, Ho S, Branch MS, Jowell PS, Baillie J, Gress FG. The Diagnostic utility of endoscopic ultrasound (EUS) and ERCP in evaluating patients with idiopathic acute recurrent pancreatitis (IARP): an update. Gastrointest Endosc. 2006; 63(5):AB262 (W1304).

77. Gregor JC, Ponich TP, Detsky AS. Should ERCP be routine after an episode of "idiopathic" pancreatitis? A cost-utility analysis. Gastrointest Endosc. 1996; 44(2):118-23.

78. Frossard JL, Sosa-Valencia L, Amouyal G, Marty O, Hadengue A, Amouyal P. Usefulness of endoscopic ultrasonography in patients with "idiopathic" acute pancreatitis. Am J Med. 2000; 109(3):196200.
79. Block MA, Priest RJ. Acute pancreatitis related to grossly minute stones in a radiographically normal gallbladder. Am J Dig Dis. 1967; 12(9):934-8.

80. Good LI, Edell SL, Soloway RD, Trotman BW, Mulhern C, Arger PA. Ultrasonic properties of gallstones. Effect of stone size and composition. Gastroenterology. 1979; 77(2):258-63.

81. Simeone JF, Mueller PR, Ferruci JT Jr., Harbin WP, Wittenberg J. Significance of nonshadowing focal opacities at cholecystosonography. Radiology. 1980; 137(1 Pt 1):181-5.

82. Goinard P, Pelissier G. [Biliary micro-lithiasis]. Presse Med. 1962; 70:260-1.

83. Farrar JT. Underdiagnosis of biliary tract disorders? Gastroenterology. 1966; 51(6):1074-5.

84. Venu RP, Toouli J, Geenen JE, Stewart ET, Hogan WJ. Migrating common bile duct stones. Dig Dis Sci. 1981; 26(10):949-53.

85. Venu RP, Geenen JE, Toouli J, Stewart E, Hogan WJ. Endoscopic retrograde cholangiopancreatography. Diagnosis of cholelithiasis in patients with normal gallbladder x-ray and ultrasound studies. JAMA. 1983; 249(6):758-61.

86. Feller ER. Endoscopic retrograde cholangiopancreatography in the diagnosis of unexplained pancreatitis. Arch Intern Med. 1984; 144(9):1797-9.

87. Aliperti G. Complications related to diagnostic and therapeutic endoscopic retrograde cholangiopancreatography. Gastrointest Endosc Clin N Am. 1996; 6(2):379-407.

88. Thoeni RF, Fell SC, Goldberg HI. CT detection of asymptomatic pancreatitis following ERCP. Gastrointest Radiol. 1990; 15(4):2915.

89. Liu CL, Lo CM, Chan JK, Poon RT, Fan ST. EUS for detection of occult cholelithiasis in patients with idiopathic pancreatitis. Gastrointest Endosc. 2000; 51(1):28-32.

90. Tandon M, Topazian M. Endoscopic ultrasound in idiopathic acute pancreatitis. Am J Gastroenterol. 2001; 96(3):705-9.

91. Levy P, Boruchowicz A, Hastier P, Pariente A, Thevenot T, Frossard $J \mathrm{~L}$ et al. Diagnostic criteria in predicting a biliary origin of acute pancreatitis in the era of endoscopic ultrasound: multicentre prospective evaluation of 213 patients. Pancreatology. 2005; 5(45):450-6.

Recebido em 19/09/2008

Aceito para publicação em 24/11/2008

Conflito de interesse: nenhum

Fonte de financiamento: nenhuma

Como citar este artigo:

Ardengh JC, Coelho DE, Santos JS, Módena JLP, Eulálio JMR, Coelho JF. Pancreatite aguda sem etiologia aparente: a microlitíase deve ser pesquisada? Rev Col Bras Cir. [periódico na Internet] 2009; 36(5). Disponível em URL: http://www.scielo.br/rcbc

Endereço para correspondência:

José Celso Ardengh

E-mail: jcelso@uol.com.br 